\title{
Mechanical behavior of fiber/matrix interfaces in CFRP sheets subjected to plastic deformation
}

\author{
Ryuta Kamiya $^{1, a}$ and Tetsuo Oya ${ }^{1}$ \\ ${ }^{1}$ Keio University, 3-14-1 Hiyoshi, Kohoku-ku, Yokohama, Kanagawa, Japan
}

\begin{abstract}
The use of Carbon Fiber Reinforced Plastic (CFRP) is increasing markedly, partially in the aviation industry, but it has been considered that CFRP sheets cannot be formed by press-forming techniques owing to the low ductility of CFRP. Since the mechanical characteristics of CFRP are dominated by the microscale structure, it is possible to improve its formability by optimizing the material structure. Therefore, to improve the formability, the interaction between the carbon fibers and the matrix must be clarified. In this study, microscale analyses were conducted by a finite-element model with cohesive zone elements.
\end{abstract}

\section{Introduction}

The demand for lower manufacturing costs, increased safety, improved fuel consumption and a shorter delivery time is increasing for transport equipment. In recent years, carbon fiber reinforced plastic (CFRP) has been focused on as one of the most promising lightweight materials that can meet these requirements. Although it has higher specific strength and higher specific stiffness than metals, CFRP has low ductility. Because of its low ductility, a forming method that can process a large number of CFRP sheets in a short time such as press forming has not been established. If a plastic working method for CFRP (particularly thermosetting CFRP) were constructed, CFRP sheets could be applied to various products. Since the macroscopic mechanical characteristics of CFRP sheets are dominated by the microscopic structure, such as the material properties of the fiber/matrix interface, the internal fiber orientations and the laminated form, it maybe possible to improve the formability of CFRP sheets by modifying the internal mechanical characteristics without changing the macroscopic shape. To improve the formability, the interaction mechanism between the fibers and the matrix must be clarified.

It has already been confirmed that the interfacial properties play a crucial role in the load transfer between the fibers and the matrix and affect the global performance of a composite structure. The effect of the interfacial properties on both the microscale and macroscale composite behavior and their relationships has been studied by various researchers. Pisanova et al.[1] determined the relationships between the micromechanical interfacial shear strength and macroscopic performance of composites by performing transverse tensile and compression shear tests on glass fiber reinforced plastics. The degree of fiber matrix adhesion has been measured using micromechanical experimental methods including single-fiber pull out, fiber push out, fiber fragmentation and microdroplet tests [2]. Nishikawa et al. [3] attempted to quantify the fracture properties (strength and toughness) of the fiber/matrix interface in composites, by considering the fragmentation process and debonding growth for two types of composite. Their method was based on numerical modeling of the microscopic damage and fragmentation process in fragmentation tests using a cohesive zone model, which is often employed to express the interfacial debonding growth in fracture mechanics [4]. However, their study discussed the interactions between the fibers and the matrix (i.e., fiber breaks, matrix plasticity and cracking, and interfacial debonding) in a single fiber composite, not a CFRP sheet, during plastic deformation. Our final goal is to elucidate mechanisms of the plastic deformation of CFRP sheets using cohesive zone elements. Therefore, the present study was designed to assess the effects of the interfacial properties on the behavior of the entire material by finite-element analyses using a microscale CFRP model to express the interfacial behavior, which is considered as one of the factors that can be modified to improve the formability of CFRP sheets.

\section{Experimental procedures}

\subsection{Uniaxial tensile test}

First, a uniaxial tensile test was performed on a unidirectional CFRP specimen to determine the properties of the fibers and the matrix for use in microstructure analyses. Carbon-epoxy prepreg layers comprising continuous carbon fibers (T700SC) and epoxy resin (\#2592) were supplied by Toray Industries

\footnotetext{
a Ryuta Kamiya: seiko48057@a5.keio.jp
} 
Inc. The thickness of each prepreg layer was $0.1 \mathrm{~mm}$ and the diameter of the carbon fibers was $5 \mu \mathrm{m}$. The weight fraction of carbon fibers in the CFRP sheet was $67 \mathrm{wt} \%$. The ply laminates of the CFRP sheet were unidirectional and parallel to the tensile direction. The CFRP sheet was composed of six prepreg layers with the same thickness, and the CFRP layers were laminated by vacuum bag molding. The dimensions of the CFRP specimen are shown in Fig.1.

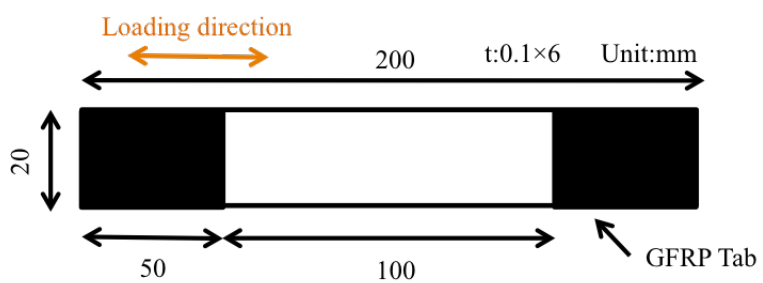

Figure 1. Dimensions of the CFRP specimen.

The tensile test was performed at room temperature using servo-hydraulic tensile testing machine with 200 $\mathrm{kN}$ capacity. The loading speed in the tensile test was fixed at $1 \mathrm{~mm} / \mathrm{min}$ up to the fracture point.

\subsection{Experimental results}

Fig. 2 and Table 1 respectively show the nominal stressstrain curve and material properties obtained from the experiment. The Young's modulus was measured in accordance with the JIS K 7161 standard.

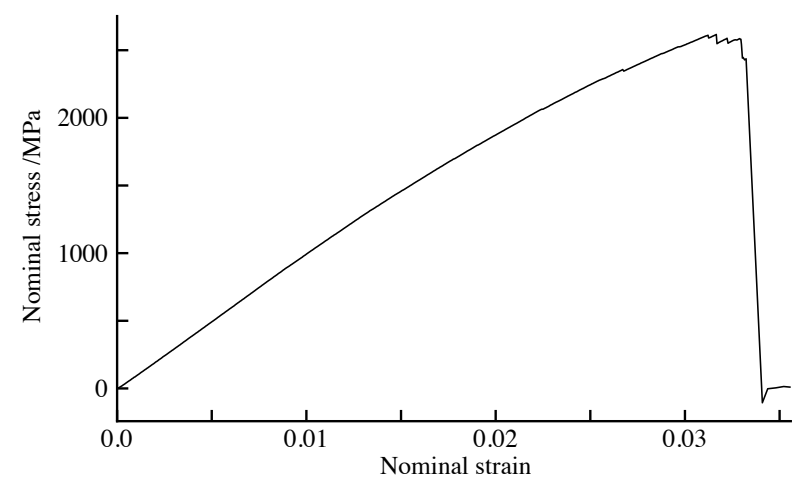

Figure 2. Nominal stress-strain curve of the specimen.

Table 1. Material properties of the specimen.

\begin{tabular}{|c|c|}
\hline Property & CFRP specimen \\
\hline Young's modulus /GPa & 99.0 \\
\hline Fracture stress $/ \mathrm{MPa}$ & 2438 \\
\hline Fracture strain & 0.033 \\
\hline
\end{tabular}

\section{Microscale analyses of the tensile test}

\subsection{Determination of material properties for use in the microscale analysis}

Fibers and resin should be modeled separately to express the interaction between the fibers and the matrix, but this is almost impossible from the view of the computational cost. Thus, a microscale unit cell model was constructed. A microscale unit cell model, which has a periodicity in three dimensions, is often used to determine the quantitative mechanical properties of CFRP subjected to elastic deformation. J. M. Guedes et al. [5] discussed the homogenization method to determine the effective average elastic constants of linear elasticity of the composite material, which is considered as the spatial repetition of a microscale unit cell model. In the homogenization method, periodic boundary conditions are often set to propagate the mechanical behavior of unit cell model. However, this study aims to observe the vicinity of a broken fiber in the entire material. Therefore, we set the boundary condition so that the opposite boundary planes are free to move but remain planer in a parallel way for assuming generality with adjacent cells.

The material properties of the fiber and the matrix were estimated by fitting them to the experimental data by the rule of mixtures. This estimation assumes that the fiber/matrix interfaces are completely fixed and that the Young's moduli of the fibers and resin follow the rule of mixture. Properties that could not be estimated from the experiment were not used in the microscale analysis. The estimated material properties are shown in Table 2.

Table 2. Material properties estimated by the rule of mixtures.

\begin{tabular}{|c|c|c|}
\hline Property & Fiber & Resin \\
\hline Young's modulus /GPa & 155.6 & 2.65 \\
\hline Poisson's ratio & 0.34 & 0.36 \\
\hline Mass density /g/cm ${ }^{3}$ & 1.80 & 1.24 \\
\hline Yield stress /MPa & - & 18 \\
\hline Tangent modulus $/ \mathrm{MPa}$ & - & 2300 \\
\hline
\end{tabular}

Next, a microscale analysis without considering interfacial behavior was performed to confirm that the estimated parameters were accurate by comparison with the elastic deformation in the experiment. Fig.3 shows the microscale model used in this section. This model was created and analyzed using the finite element code LSDYNA.

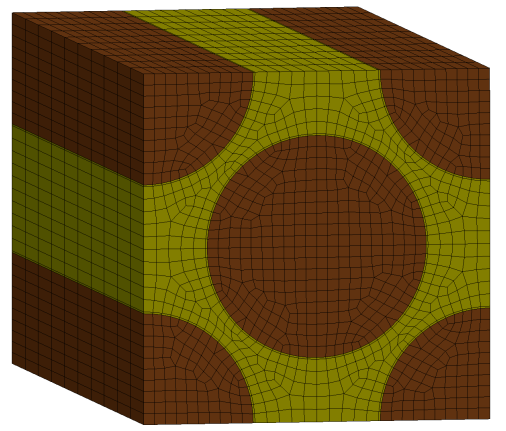

Figure 3. Microscale model of the CFRP specimen without considering interfacial behavior. 
The whole model was meshed with eight-node hexahedral solid elements. The interface between the fibers and the matrix was assumed to be well bonded. The circular parts in Fig.3 are fibers. Although fibers are normally orthotropic elastic materials, they are modeled as an isotropic elastic material in this study. The resin was modeled as an isotropic elastic-plastic material. The load in this simulation was applied by controlling the displacement of the surface perpendicular to the fiber direction and fixing the opposite surface until the nominal strain of the model reached 0.015. Fig.4 shows a comparison of the results of the experiment and the microscale analysis without considering interfacial behavior.

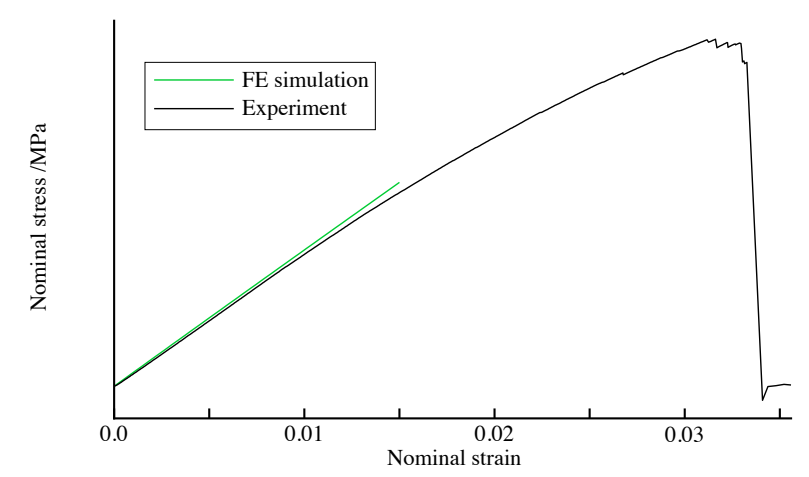

Figure 4. Comparison of the results of the experiment and microscale analysis without considering interfacial behavior.

It was found that the stress-strain curve obtained in the simulation was almost identical to that obtained in the experiment under elastic deformation. Therefore, the material properties shown in Table 2 were used in the analyses considering interfacial behavior.

\subsection{Microscale analyses by modeling with cohesive zone elements}

\subsubsection{Cohesive zone elements}

To express the fiber/matrix interfacial debonding, the application of cohesive zone elements (CZEs) to the interface was proposed. The analysis of fracture development has changed considerably in the last few decades. Instead of the traditional approaches of fracture mechanics, various predictive schemes such as CZEs are now increasingly being used, particularly in numerical simulations. The use of CZEs is a relatively simple method, making it easier to implement in finite-element codes and, as a result, CZEs are now available in various commercial FE packages such as LS-DYNA used in this simulation.

One of the first studies of cohesive zones was performed by Dugdale [6], who established the fundamental idea of CZEs. In his study, a plastic region was set around a crack tip and the relationship between the extent of plastic yielding and the applied external load was obtained. Needleman [7] was one of the first to use polynomial and exponential traction-separation relations to simulate the particle debonding in metal matrices. An exponential fit was used for a normal traction while a trigonometric fit was used for a shear traction. In short, CZEs have been found to be very useful for approaching problems involving nonlinear interfacial behavior.

This study includes the implementation of CZEs based on a bilinear traction-separation law. The mechanical response of the interface is described by constitutive equations, which relate the stresses $t$ and $s$, normal and tangential to the interface, respectively, to the relative displacements in the same directions at the interface $\delta$ by a bilinear relationship. Fig.5 shows the traction-separation law defined in this simulation. The cohesive zone model used in this study defines the same traction-separation law in the normal and tangential directions.

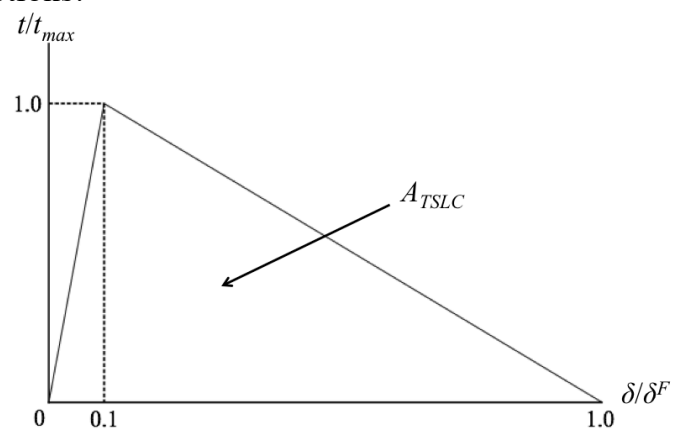

Figure 5. Normalized traction-separation law of CZEs.

This figure indicates that interfacial debonding starts at $t_{\max }$ and ends at $\delta_{\max }$. The area between the curve and the horizontal axis represents $A_{T S L C}$, which is the normalized energy release rate. Interfacial properties are determined by the energy release rate $G_{\mid}^{\mathrm{c}}$ (mode $\mid$ ), the maximum normal stress $T$ in the normal direction (mode I ), the energy release rate $G_{\|}^{\text {c }}$ (mode $\|$ ) and the maximum normal stress $S$ in the tangential direction (mode II) as follows.

$$
\delta_{\mathrm{I}}^{\mathrm{F}}=\frac{G_{1}^{\mathrm{c}}}{A_{T S L C} T}, \quad \delta_{\|}^{\mathrm{F}}=\frac{G_{\|}^{\mathrm{c}}}{A_{T S L C} S}
$$

\subsubsection{Conditions of microscale analyses with CZEs}

In this section, analyses of the tensile test with CZEs are discussed, where it was assumed that the central fiber in the microscale model ruptured at a strain of 0.015 . The effect of the properties of the cohesive zone on the fiber/matrix interfacial behavior after the breakage of a fiber was examined. Fig.6 shows the model constructed for this simulation.

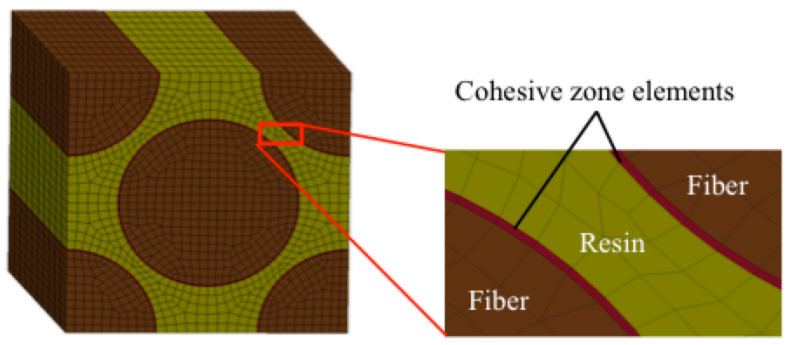

Figure 6. Microscale model with CZEs.

CZEs were inserted between the fibers and the resin. The other geometric conditions were the same as those in 
the model in section 3.1. To simulate the process of fiber breakage in the composite, a load was applied as follows. First, a uniform tensile displacement in the fiber direction was imposed on the entire top surface of the model until the nominal strain reached 0.015 as in section 3.1. After obtaining the applied composite stress, the nodes on the surface of the center fiber was released and become traction-free, thus simulating a fiber break. A tensile load was applied until the fracture strain observed in the experiment was reached. This simulation was performed for the four sets of cohesive zone properties shown in Table 3.

Table 3. Material properties of CZEs.

\begin{tabular}{|c|c|c|c|c|}
\hline Set & $G_{\mid}^{\mathrm{c}} / \mathrm{N} / \mathrm{mm}$ & $T / \mathrm{MPa}$ & $G_{\|}^{\mathrm{c}} / \mathrm{N} / \mathrm{mm}$ & $S / \mathrm{MPa}$ \\
\hline$(1)$ & 0.645 & 86 & 0.51 & 34 \\
\hline$(2)$ & 1.29 & 86 & 0.51 & 34 \\
\hline$(3)$ & 0.645 & 86 & 0.765 & 34 \\
\hline$(4)$ & 0.645 & 86 & 0.51 & 68 \\
\hline
\end{tabular}

One parameter was varied from the basic model (1) in each set of parameters with $G_{\mid}^{\mathrm{c}}, G_{\|}^{\mathrm{c}}$ and $S$ changed in sets (2) to (4), respectively. Actually, interfacial properties should have been determined by characteristic tests. However, we used estimated values instead because it is extremely difficult to measure cohesive zone properties in experiments. Therefore, we qualitatively discuss the effect of changing the interfacial parameters.

\subsection{Analytical results of interfacial debonding}

\subsubsection{Effect of the energy release rate normal to the fibers}

The results for sets (1) and (2) were compared to observe the effect of $G_{\mid}^{\mathrm{c}}$. Fig.7 shows a comparison of the normal-direction stress-strain curve of the CZEs at specified distances from the fiber break point. Points with zero stress in the figure indicate complete debonding at corresponding distance.

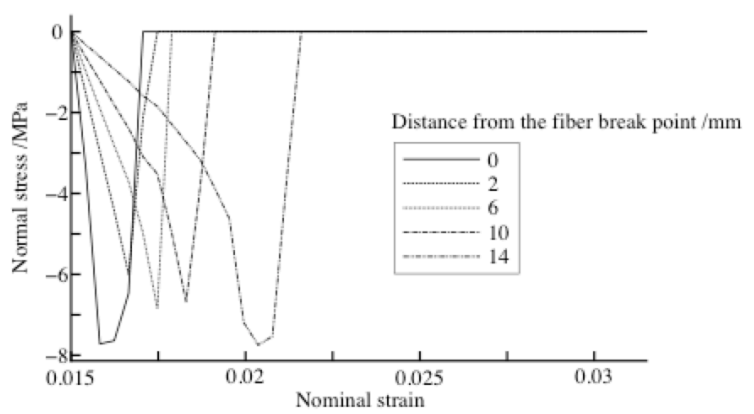

(a)

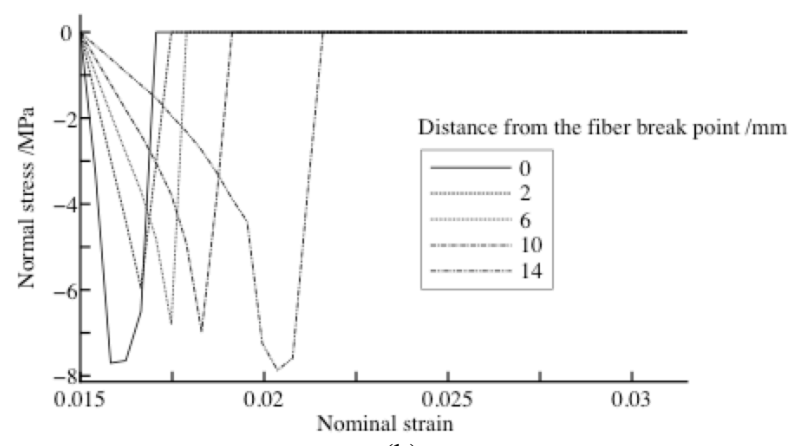

(b)

Figure 7. Normal-direction stress-strain curves of CZEs at specified distances from the fiber break point: (a) set (1) and (b) set (2).

By considering the complete debonding points, it was found that no major differences occurred upon changing the value of $G_{1}^{\mathrm{c}}$. This result clearly shows that the result of a tensile test on unidirectional CFRP sheets does not depend on the fiber/matrix interfacial characteristics normal to the fibers.

\subsubsection{Effect of the energy release rate tangential to the fibers}

The results for sets (1) and (3) were compared to observe the effect of $G_{\|}^{\mathrm{c}}$. Fig.8 shows a comparison of the tangential-direction stress-strain curve of the CZEs at specified distances from the fiber break point.

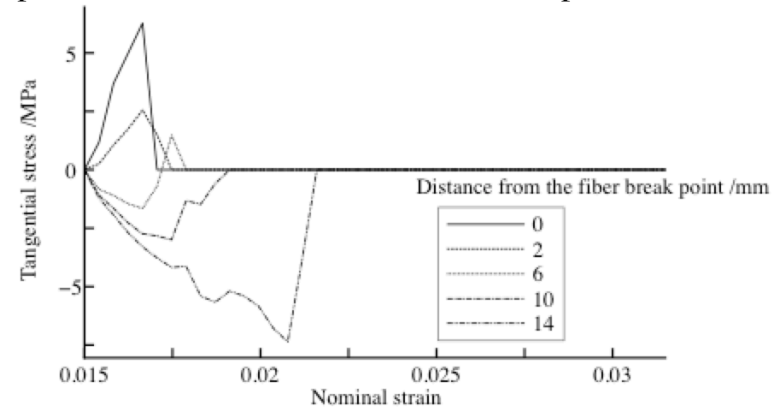

(a)

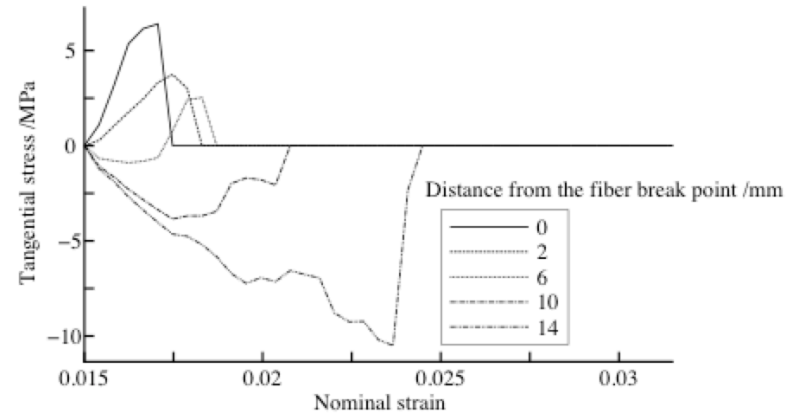

(b)

Figure 8. Tangential-direction stress-strain curves of CZEs at specified distances from the fiber break point: (a) set (1) and (b) set (3).

Fig.8 indicates that the complete debonding strain of set (3) is larger than that of set (1) at all distances. It can be concluded that the tangential-direction energy release rate $G_{\|}^{\text {c }}$ substantially dominates the degree of resistance to interfacial debonding. 


\subsubsection{Effect of the maximum stress tangential to the fibers}

The results for sets (1) and (4) were compared to observe the effect of the maximum shear stress $S$. Fig.9 shows a comparison of the tangential-direction stress-strain curve of the CZEs at specified distances from the fiber break point.

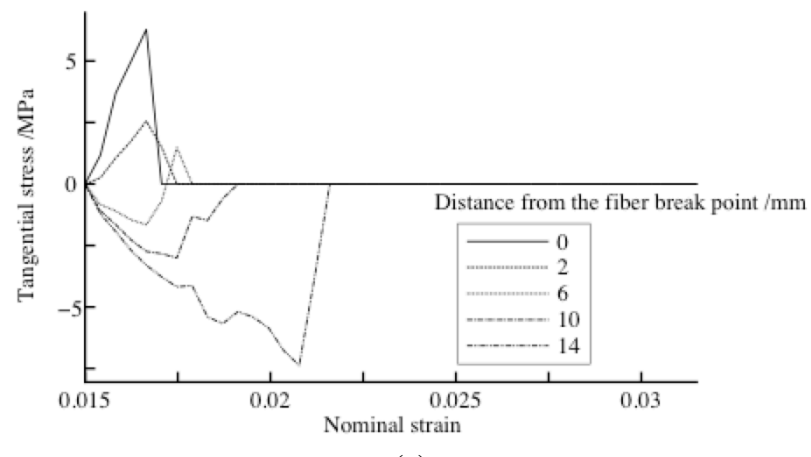

(a)

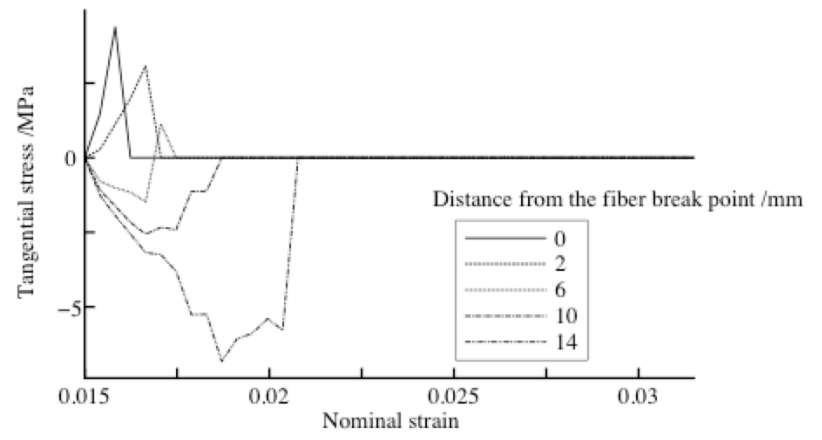

(b)

Figure 9. Tangential-direction stress-strain curves of CZEs at specified distances from the fiber break point: (a) set (1) and (b) set (4).

Fig.9 shows that the interfacial debonding of set (4) ends at a lower strain. This indicates that an increase in the tangential maximum stress makes it easy to debond the fiber/matrix interface.

\subsection{Analytical results of the stress distributions around the fibers}

In general, a CFRP breaks as a result of local stress concentrations caused by a complicated mixture of internal fiber breakage, interfacial debonding and matrix cracking. Thus, understanding the stress distribution around a fiber is also important for investigating the mechanism of plastic deformation for CFRP sheets. Fig.10 shows the stress distributions of the bottom surface of the resin for each set after the simulation.

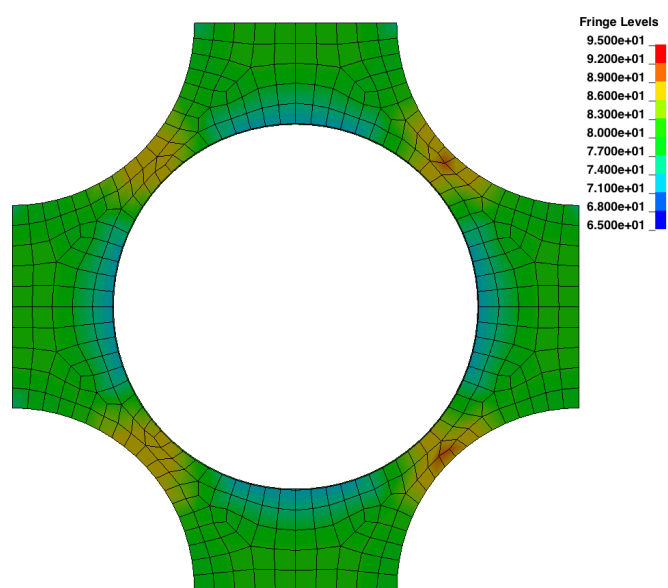

(a)
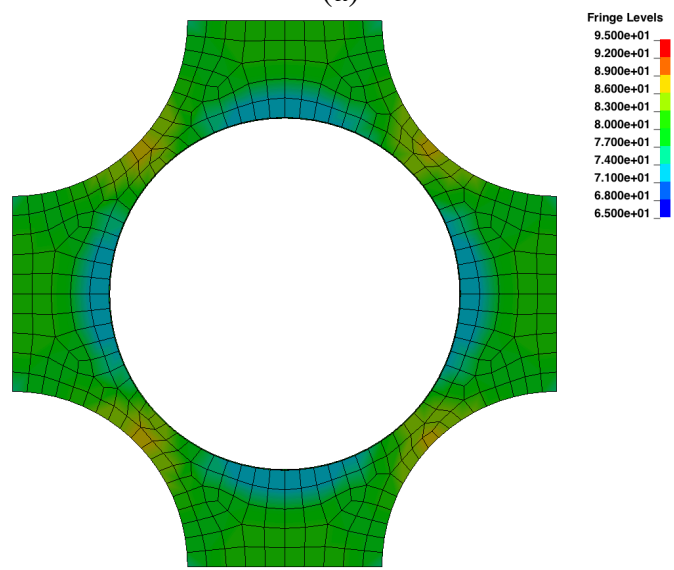

(b)

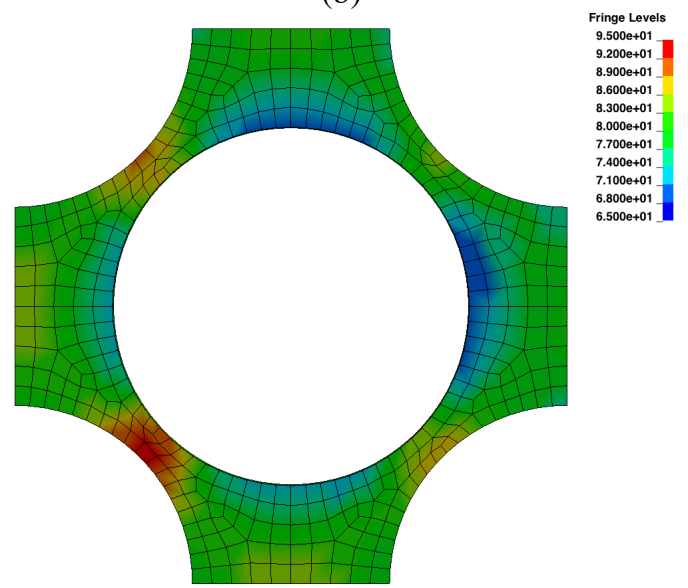

(c)

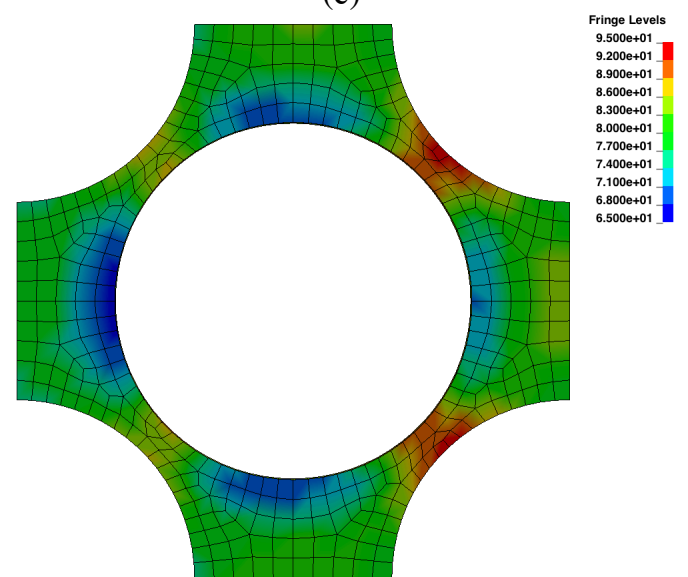

(d)

Figure 10. Stress distributions of the bottom surface of the resin: (a) set (1), (b) set (2), (c) set (3) and (d) set (4). 
Fig.10 (d), which was obtained at a large maximum tangential stress, shows the most scattering of the stress distribution among the sets. In other words, the cohesive zone property of set (4) tends to concentrate the local stress. This is consistent with the experimental result that composites have a stronger fiber/matrix interface, making matrix cracking around the fiber break point more likely to occur [3]. Therefore, the CZEs in this model qualitatively well express the fiber/matrix interfacial behavior.

\section{Conclusion}

A uniaxial tensile test on a CFRP specimen and microscale analyses were performed. Fibers and matrix properties were estimated by the rule of mixtures for composites using the experimental results.

CZEs were applied to the fiber/matrix interfaces in the microscale unit cell model. Analytical results indicated that the properties of cohesive zone elements qualitatively well represented the actual fiber/matrix interfacial behavior. This result will be helpful in understanding and modeling the deformation processes in CFRP sheets.

As a future task, a means of identifying quantitative interfacial properties under various thermal environments and considering other interactive factors, such as the friction after debonding or the viscosity of the resin, should be established using CZEs.

\section{References}

1. E. Pisanova, S. Zhandarov, E. Mader, I. Ahmad, R. J. Young, Composites Part A 32, 435 (2001).

2. M. J. Pitkethly, J. P. Favre, U. Gaur, J. Jakubowski, S. F. Mudrich, D. L. Caldwell, L. T. Drzal, M. Nardin, H. D. Wagner, L. Di Landro, A. Hampe, J. P. Armistead, M. Desaeger, I. Verpoest, Compos. Sci. Technolo. 48, 205 (1993).

3. M. Nishikawa, T. Okabe, N. Takeda, Mater. Sci. Eng. A 480, 549 (2008).

4. B. W. Kim, J. A. Nairn, J. Compos. Mater. 36, 1825 (2002).

5. J. M. Guedes, N. Kikuchi, Comput. Methods in Appl. Mech. Eng. 83, 143 (1990).

6. D. S. Dugdale, J. Mech. Phys. Solids 8, 100 (1960).

7. A. Needleman, J. Mech. Phys. Solids 38, 289 (1990). 